\title{
Interpreting confidence intervals: A comment on Hoekstra, Morey, Rouder, and Wagenmakers (2014)
}

\author{
Jeff Miller ${ }^{1} \cdot$ Rolf Ulrich ${ }^{2}$
}

Published online: 11 November 2015

(C) Psychonomic Society, Inc. 2015

\begin{abstract}
Hoekstra, Morey, Rouder, and Wagenmakers (Psychonomic Bulletin \& Review 21(5), 1157-1164 2014) reported the results of a questionnaire designed to assess students' and researchers' understanding of confidence intervals (CIs). They interpreted their results as evidence that these groups "have no reliable knowledge about the correct interpretation of CIs" (Hoekstra et al. Psychonomic Bulletin \& Review 21(5), 1157-1164 2014, p. 1161). We argue that their data do not substantiate this conclusion and that their report includes misleading suggestions about the correct interpretations of confidence intervals.
\end{abstract}

Keywords Confidence intervals $\cdot$ Statistical inference

\section{Background}

In the interests of scientific progress, researchers should continually re-examine both the methods they use and their understanding of those methods. At present, it seems especially appropriate to examine researchers' conceptions of statistical techniques, because there is evidence of statistically questionable research practices (QRPs; e.g., Ioannidis, 2005; Simmons et al., 2011), leading to concerns about the

Jeff Miller

miller@psy.otago.ac.nz

Rolf Ulrich

ulrich@uni-tuebingen.de

1 Department of Psychology, University of Otago, PO Box 56, Dunedin 9054, New Zealand

2 Department of Psychology, University of Tübingen, Schleichstr. 4, Tübingen 72076, Germany replicability of published findings (e.g., Carpenter, 2012; Francis, 2013; Open Science Collaboration, 2012; Pashler and Harris, 2012; Simonsohn, Nelson, and Simmons 2014). Studies documenting statistical misconceptions can be especially worthwhile by serving the dual roles of sounding an alarm that more education is needed and of providing that education. At the same time, however, the results of such studies must be interpreted cautiously if the overall research enterprise is to be advanced. Sounding exaggerated alarms may create confusion and distract researchers from substantive issues that they could otherwise investigate.

One recent study of statistical understanding was reported by Hoekstra et al. (2014) (henceforth, HMRW), who investigated researchers' understanding of confidence intervals (CIs). HMRW presented a questionnaire to a sample including 442 bachelor students taking an introductory statistics class, 34 master students, and 120 researchers, with the latter two groups expected to be well informed about statistical matters. Participants were instructed to imagine that a study had been conducted, that the data were used to compute a $95 \% \mathrm{CI}$ for the population mean, $\mu$, and that the resulting CI ranged from 0.1 to 0.4 . The questionnaire included six statements involving this CI (see Table 1), and participants were instructed to indicate whether each of the six statements was true or false. According to HMRW, all six statements are false, and "Researchers who are aware of the correct interpretation of a CI should have no difficulty checking all 'false' boxes" (p. 1160). In fact, students and researchers alike endorsed more than half of these statements as true. From that finding, HMRW reached a strong negative conclusion: "one might have expected that most researchers in psychology would be well informed about the interpretation of this rather basic inferential outcome. Our data, however, suggest that the opposite is true: Both researchers and students in psychology have 
Table 1 The six statements on the questionnaire of Hoekstra et al. (2014)

1. The probability that the true mean is greater than 0 is at least $95 \%$.

2. The probability that the true mean equals 0 is smaller than $5 \%$.

3. The "null hypothesis" that the true mean equals 0 is likely to be incorrect.

4. There is a $95 \%$ probability that the true mean lies between 0.1 and 0.4 .

5. We can be $95 \%$ confident that the true mean lies between 0.1 and 0.4 .

6. If we were to repeat the experiment over and over, then $95 \%$ of the time the true mean falls between 0.1 and 0.4 .

Participants were told to imagine that a hypothetical study had resulted in a confidence interval for a mean ranging from 0.1 to 0.4 and to indicate whether based on that study each of these six statements was true or false

no reliable knowledge about the correct interpretation of CIs" (Hoekstra et al., 2014, p. 1161). On the basis of these findings, as well as a rather artificial case in which a debatable application of CIs seems to produce inappropriate results (e.g., DeGroot 1989; Robinson 1975), these authors subsequently suggested that CIs should essentially be abandoned (Morey, Wagenmakers, Hoekstra, Rouder \& Lee 2014).

\section{Goals of this article}

In psychology, CIs are most often used to summarize information about measurement error (e.g., Cumming and Finch 2005) and thereby to facilitate the interpretation of both differences between observed means or proportions and differences between observed values and values predicted by a model. These CIs have a solid mathematical foundation (Neyman, 1937) that has not been disputed, even by critics (e.g., Robinson 1975), suggesting that they could be very useful data analytic tools. Indeed, a task force set up to examine statistical procedures in psychology recommended that researchers should increase their use of CIs (Wikinson \& the task Force on Statistical Inference, 1999). Given that CIs have these positive features, it seems worthwhile to reexamine HMRW's arguments that they are poorly understood and should be avoided.

In this article, we argue that HMRW's study is misleading in two ways. First, it may underestimate the extent to which researchers understand CIs. As will be discussed in detail, statements 1-4 of Table 1 are incompatible with a "strict frequentist" interpretation of the word "probability", but participants may have had a different interpretation of that word in mind when completing the questionnaire. In that case, their acceptance of these statements as true may not necessarily indicate misunderstanding of CIs per se, but rather use of a different interpretation of "probability."

Second, HMRW's conclusions are likely to leave many readers with confusion or misconceptions about how CIs actually should be interpreted. Statement 5 in Table 1, which HMRW claim to be false, is essentially the standard interpretation of CIs recommended by authoritative statistics texts. As is discussed in the section "What is a confidence interval?", somewhat more elaborate correct interpretations of CIs can also be given, and these help to further clarify the meanings of these intervals.

Our primary goals in this note are educational. In the section "What is a confidence interval?," we review the fundamental logic and interpretation of CIs. In the section "Why isn't the probability $95 \%$ ?," we explain in detail why statements 1-4 are incompatible with a strict frequentist definition of "probability" but might nonetheless be regarded as appropriate under other meanings of "probability" that are in common use. An appreciation of this distinction will not only improve researchers' understandings of CIs but will also make clear why HMRW's questionnaire results should not be regarded as evidence of serious misunderstandings. Finally, in the section "Can we be $95 \%$ confident?," we will show that HMRW's statement 5 is actually a very standard interpretation of a CI; in some texts, it is a kind of short-hand for the definition of a CI.

\section{What is a confidence interval?}

Within mathematical statistics, a CI is always derived as a random interval. This random interval is defined by two formulas, here denoted $A$ and $B$, which can be shown to have a certain known long-run probability of capturing the parameter of interest when the intervals are computed across many random samples ${ }^{1}$. In the case of the $\mathrm{CI}$ for the mean $\mu$ of a normally distributed population ${ }^{2}$, for example, the standard formulas for $A$ and $B$ are

$$
\begin{aligned}
& A=\bar{X}-\frac{S}{\sqrt{n}} \cdot t_{.975, n-1} \\
& B=\bar{X}+\frac{S}{\sqrt{n}} \cdot t_{.975, n-1}
\end{aligned}
$$

where $n, \bar{X}$, and $S$ are the sample size, mean, and standard deviation, respectively, and $t_{975, n-1}$ is the two-tailed

\footnotetext{
${ }^{1}$ Typically, these formulas must satisfy other technical criteria as well, such as defining the shortest possible interval (DeGroot, 1989, p. 338), but these criteria have no relevance to the present discussion.

${ }^{2}$ Like HMRW, we focus on $95 \%$ CIs for means to simplify the discussion and because these are the most commonly used CIs. With minor modifications to suit the circumstances, however, all of the points made in this article apply equally well to other levels of confidence (e.g., $99 \%$ ) and to confidence intervals for parameters other than means (e.g., proportions).
} 
critical value from a $t$ distribution with $n-1$ degrees of freedom (e.g., Hays, 1973). Because these formulas can be applied to any random sample, $\bar{X}$ and $S$ are random variables and thus the interval $(A, B)$ is a random interval with the desired feature that-across a large number of random samples-95\% of the computed intervals will contain $\mu$.

Using formulas such as $A$ and $B$ provided by mathematical statisticians, researchers in practice use their data to compute fixed CIs having known numerical end points, denoted here as $a$ and $b$. For example, with a sample of $n=20$ cases having $\bar{X}=0.25$, and $S=0.32$, the researcher would use Eqs. 1 and 2 to compute $a=0.1$ and $b=0.4$, yielding the fixed $95 \%$ CI of $0.1-0.4$. Crucially, the true value of $\mu$ is also a fixed number, and it either lies within this interval or it does not. That is, the interval $0.1-$ 0.4 may be one of the $95 \%$ of fixed CIs that contain $\mu$, or it may be one of the $5 \%$ that do not, and there is typically no way to be sure which of these is the case for any particular fixed CI.

What is the correct interpretation of a fixed CI such as 0.1-0.4? The correct conclusion suggested by HMRW is "If we were to repeat the experiment over and over, then $95 \%$ of the time the confidence intervals contain the true mean" (Hoekstra et al., 2014, p. 1160). This statement, although formally correct, does not seem entirely satisfactory because it does not mention the bounds 0.1 and 0.4 that were actually obtained from the analysis of the data. Any interpretation of sample data should in some way summarize the information provided by the sample.

An appropriate conclusion concerning a confidence interval-analogous to statement 4 but elaborated to clarify the role of probability-could take the following form ${ }^{3}$ :

4'. If the current sample is one of the $95 \%$ of all samples with relatively small values of $|\bar{X}-\mu| / S$, then $\mu$ lies in the interval $0.1-0.4$.

Equivalently, the conclusion's premise could be phrased as "Unless the current sample is one of the $5 \%$ of all samples with unusually large values of $|\bar{X}-\mu| / S, \ldots$ "

Conclusions of this form include an explicit statement making clear the degree of confidence and its mathematical justification (i.e., $\mu$ lies within the CI for $95 \%$ of all samples). Usefully, they also convey the sample information by stating the computed numerical bounds. Moreover,

\footnotetext{
${ }^{3}$ Although it is common to judge deviations $\bar{X}-\mu$ relative to the standard error, $S / \sqrt{n}$, rather than relative to the standard deviation, $S$, we used the simpler expression because it conveys exactly the same information about which samples result in larger deviations.
}

MHRW's participants may have had just such interpretations in mind when they endorsed statement 4. Note that the word "probability" is conspicuously absent from these interpretations. As is explained in the next section, much of the confusion surrounding the interpretation of CIs can be traced to inconsistencies in the use of this multifaceted word (e.g., Hájek, 2012). Fortunately, the strong mathematical foundations of CIs provide them with clear interpretations (e.g., 4') that do not use the word "probability" and thus do not rely on any particular meaning of that word.

\section{Why isn't the probability $95 \%$ ?}

It is important to consider in detail why statement 4 is incompatible with a strict frequentist interpretation of "probability." As HMRW see it, "Statements 1, 2, 3, and 4 assign probabilities to parameters or hypotheses, something that is not allowed within the frequentist framework" (p. 1160). Dracup (2005) provides a more elaborate explanation (for similar explanations, see e.g., DeGroot, 1989; Hogg \& Craig 1970):

...for any particular interval that has been calculated, it will be either one of the $95 \%$ that contain the population mean or one of the $5 \%$ that do not. There is no way of knowing which of these two is true. According to the frequentist view of probability... for any particular ["fixed"] confidence interval the population [sic] is either included or not included in it. The probability of inclusion is therefore either 1 or 0 . The confidence interval approach does not give the probability that the true population mean will be in the particular interval constructed (Dracup, 2005, p. 367, italicized portion added).

This argument has two critical ingredients. First, according to a strict frequentist interpretation, the probability of something is its long-run frequency of occurrence within a series of independent random outcomes. Second, when considering the particular fixed confidence interval $0.1-0.4$, there is no long-run series of random events. The endpoints of this fixed interval are the constant numerical values 0.1 and $0.4 . \mu$ is also a constant, even though its precise value is unknown. Thus, the CI statement " $0.1 \leq \mu \leq 0.4$ " is a specific isolated numerical assertion, and it is either true or false (i.e., $\mu$ either lies between 0.1 and 0.4 or it does not). When the statement is viewed in this fashion, the concept of "in the long run" hardly seems to apply, since the truth value does not fluctuate. If forced to assign it a probability, a strict frequentist could only say that its "probability ... is ... either 1 or 0” (Dracup, 2005, p. 367)—not $95 \%$. 
Gilliland and Melfi (2010) (2010, p. 2) nicely explain the implications of this argument for the interpretation of obtained numerical bounds:

There is a certain awkwardness in interpreting the interval once the data are available and the endpoints are calculated. Returning to the above example, suppose that $n=25, \sigma=10$ and the simple random sampling process results in a sample with mean $\bar{y}=50$. The realized interval estimate is $50 \pm 4$. Many writers tell the readers that a statement like $\operatorname{Pr}(46<$ $\mu<54)=0.954$ is to be avoided since " $\mu$ is either in the interval $(46,54)$ or not." This reluctance is natural to the frequentist since the statement $\operatorname{Pr}(46<\mu<54)$ $=0.954$ might suggest that there is a probability distribution over the parameter values [i.e., that $\mu$ varies randomly]. Therefore, we refer to a level of confidence $95.4 \%$ for the interval $46<\mu<54$.

Even though statement 4 is incompatible with a strict frequentist interpretation in which the current $\mathrm{CI}$ is regarded as a single isolated instance, we find little cause for concern in HMRW's finding that many of their participants regarded it as true. The strict frequentist definition of "probability" does have strong advocates (e.g., Von Mises 1957), but it is only one of the many meanings of that term with significant philosophical precedent (e.g., Chatterjee, 2003; Hajek, 2012). Indeed, according to (Savage, 1954), "as to what probability is and how it is connected with statistics, there has seldom been such complete disagreement and breakdown of communication since the Tower of Babel" (p. 2). Thus, it would not be surprising if some of HMRW's participants interpreted statement 4 using some other meaning of "probability."

When answering questions about probability, people often implicitly consider the long-run frequencies of the various outcomes of some random process, even when the question refers to a particular outcome. In support of this claim, we offer the following easily-replicated observation. In discussing the meaning of "probability" with students and colleagues, we sometimes flip a coin, cover the result, and ask, "what is the probability that the coin is heads?" Everyone answers $50 \%$, indicating that they think of the question in terms of a long-run sequence of many possible flips. No one answers "it either is heads or it isn't" or "it is either heads with probability 1 or tails with probability 1", which would be the strict frequentist answers when thinking about the flip as a single isolated event. Considering probability from that long-run perspective, participants may have interpreted statement 4 as having the meaning given by our elaborated statement 4'. In that case, it is no surprise that they would endorse the statement.
A further simple and concrete example helps to illustrate our point. Suppose that a standard deck of 52 playing cards was shuffled and placed on a table at 11:00 a.m., and then it was shuffled again at 11:05, with no one having examined it between shuffles. Then, at 11:05, you are asked whether it is true or false that "There is a $1 / 52$ probability that the top card was the ace of spades at 11:01." If you are a strict frequentist, you must say this statement is false, because technically it refers specifically to the specific state of the deck at that time. As a matter of logic, the top card at 11:01 either was the ace of spades or it was not. If you had checked this card over and over-without reshuffling the deck, of course, since the statement concerns its state "at 11:01"-you would always have gotten the same result, so in the long run across these many redundant checks the probability would be 0 or 1 .

Nevertheless, we think most people would say there was a 1/52 probability that the top card was the ace of spades at 11:01, because they do not think of a specific state of the deck as a single isolated outcome in the strict frequentist sense. Implicitly, they would interpret the probability statement as referring to a long-run scenario involving many shuffled decks of cards-not just to one specific isolated deck. When the question is viewed in terms of this long-run scenario, it is true that the ace of spades will be on top in $1 / 52$ of the decks, and so it is correct to say that the probability of this event is $1 / 52$ within this long-run scenario. This example illustrates that in common parlance the word "probability" is often taken to involve an implicit series of random events, especially when talking about random outcomes that are unknown-even if they have already been determined, like the identity of the top card in the shuffled deck. Computer random number generators (RNGs) provide another good illustration of this terminology; once the seed for the RNG is chosen, the entire random sequence is determined, and yet it is standard to make statements about the probability that the next number will have a certain value. Indeed, there is even some precedent for this usage among frequentists, with the terms "predictive probability" and "postdictive probability" suggested as a way to highlight the distinction between probability statements made before a random event has taken place and statements made after the event has taken place but before its outcome is known (Dempster, 1964).

Most critically, suppose a person does assert-contrary to the strict frequentist conception of probability- "There is a $1 / 52$ probability that the top card is the ace of spades." Should we conclude that this person has "no reliable knowledge" (Hoekstra et al., 2014, p. 1161) about probability, decks of cards, shuffling, or the conclusions that can be drawn within these probabilistic scenarios? We think not. A more plausible interpretation is that the person has just 
used "probability" in its long-run, postdictive sense, ignoring the fact that the statement under consideration has now already been determined. Furthermore, there is no reason to think that the person will form an incorrect opinion, reach an incorrect decision, or take an incorrect action by relying on the long-run conception of probability in this case. Similarly, many of HMRW's participants may have interpreted the word "probability" in this long-run sense when they considered statement 4 of the questionnaire ${ }^{4}$. In that case, their acceptance of statement 4 should not be regarded as evidence that they misunderstand CIs - only that they misunderstood the researchers' intended technical meaning of "probability."

Probability statements about unobserved cards in a shuffled deck are analogous to those about computed confidence intervals. In both cases a random process generates a result (a confidence interval that does or does not contain $\mu$, a deck that does or does not have the ace of spades on top), but the actual result is unknown. Based on plausible assumptions about the nature of the random process, probability theory can be used to make statements about the expected long-run frequencies of the different outcomes. Whether one wants to make parallel statements about the "probability" of an isolated-but-unknown previously determined outcome is a matter of terminology rather than a factual matter. Assuming that terminology should be used consistently, though, anyone who says "there is a $1 / 52$ probability that the top card was the ace of spades at 11:01" should surely also say "there is a .95 probability that $\mu$ lies in the interval $0.1-0.4$ ", even though neither statement adheres to the strict frequentist approach. When making the latter statement, though, it must be kept in mind that the random process determines the bounds of the numerical interval—not the value of $\mu$.

\section{Can we be $95 \%$ confident?}

We turn now to statement 5 in Table 1. HMRW argue that this statement is false because it "mention[s] the boundaries of the CI (i.e., 0.1 and 0.4), whereas, as was stated above, a CI can be used to evaluate only the procedure and not a specific interval" (p. 1160). By this argument, a correct interpretation of a $\mathrm{CI}$ can never mention the numerical bounds of the $\mathrm{CI}$ that are computed from the sample, but such an argument is clearly inconsistent with the stated purpose of CIs: "We can then tell the practical statistician that $\ldots$ he may estimate [ $\mu$ as follows:] ... (a) he must perform the random experiment and observe the particular values $x_{1}$, $x_{2}, \ldots$ of the $X$ 's; (b) he must use these values to calculate the corresponding [lower and upper fixed numerical bounds

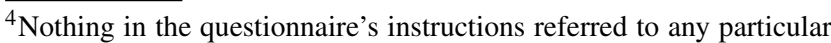
definition or interpretation of the word "probability."
}

$a$ and $b]$; and (c) he must state that $[a<\mu<b]$, where $[\mu]$ denotes the true value" (Neyman, 1937, p. 348).

In fact, HMRW's statement 5 appears to be essentially the standard interpretation of a confidence interval under a strict frequentist definition of "probability," as is illustrated by the following three quotations ${ }^{5}$. Each of these quotations occurs within a passage reiterating the strict frequentist interpretation of "probability" that was discussed in the previous section, and all of them clearly suggest "confidence" as an alternative term to use when interpreting the fixed intervals.

1. "The probability that the random interval... covers the unknown true mean $\mu$ is $.95 \ldots$. We therefore have considerable confidence that the observed interval... covers the true mean. The measure of our confidence is 95 because before the sample was drawn .95 was the probability that the interval that we were going to construct would cover the true mean" Mood, Graybill, and Boes (1974), p. 375.

2. "[after the sample is observed] we say that we have $95 \%$ confidence that $[0.1] \leq \mu \leq[0.4] \ldots$ or that $[0.1-0.4]$ is a $95 \%$ confidence interval for $\mu$ " (Robinson, 1982, p. 121).

3. "After the values... in the random sample have been observed, the values of $A$ and $B$ can be computed. If these values are $A=a$ and $B=b$, then the interval $(a, b)$ is called a confidence interval for $\mu$ with confidence coefficient 0.95 . We can then make the statement that the unknown value of $\mu$ lies in the interval $(a, b)$ with confidence 0.95." (DeGroot, 1989, p. 337)

It seems clear that the authors of these quotations would have accepted HMRW's statement 5 as an appropriate conclusion from the given sample. If so, then by HMRW's argument, they must not understand CIs either. That is logically possible, but it is implausible that such well-established mathematical statisticians do not understand CIs. Instead, these mathematical statisticians may have in mind a different interpretation of statement 5 than the one that HMRW regard as incorrect. This is important, because HMRW's participants may have interpreted statement 5 in the same way as the mathematical statisticians. If so, then their agreement with the statement need not indicate misunderstanding of CIs, contrary to HMRW's conclusion.

For interpreting statement 5 , the critical question is what it means to be "95\% confident". Unfortunately, neither the

\footnotetext{
${ }^{5}$ In quotation 2 , we have replaced the numerical values of the original author's example with the numerical values of 0.1 and 0.4 from HMRW's example.
} 
authors of the quotations nor HMRW say exactly what they mean by that, nor do we know how HMRW's participants interpreted it. For the authors of the quotations, saying that we are "95\% confident" of a statement appears to be just a compact way of saying, "the statement is a random selection from a population of statements that are known to be $95 \%$ accurate overall, and we have no other basis on which to judge the accuracy of the statement." Furthermore, using the term "confidence" in this way seems quite reasonable, since it means that confidence and accuracy will be well calibrated in the long run (e.g., Keren 1991). The earlier example involving a shuffled deck of cards provides a particularly transparent illustration. As noted earlier, after the deck was shuffled, a strict frequentist would say there was not a $1 / 52$ probability that the top card was the ace of spades. Even so, it seems quite reasonable to say that he or she had $1 / 52$ confidence that this was the top card, and it would be interesting to know why HMRW would regard such a statement as false.

\section{Conclusions}

Our two main goals have been to argue-contrary to HMRW's assertions-that participants' agreement with statement 4 cannot unambiguously be taken as a sign of misunderstanding CIs and that statement 5 is actually a very appropriate conclusion from a CI. Broadening the argument slightly, we would like to emphasize that the same points we made about statement 4 could also be made about statements 1-3. Like statement 4, HMRW regard statements 1-3 as false because they all "assign probabilities to parameters" (HMRW, p. 1160). Nonetheless, it is possible to state similar yet entirely appropriate conclusions using something like the statement 4' that we recommended above. For example, here are approximate equivalents for their statements (1) and (3):

$1 '$ If the current sample is one of the $95 \%$ of all samples with relatively small values of $|\bar{X}-\mu| / S$, then the true mean is greater than 0 .

$3^{\prime}$. If the current sample is one of the $95 \%$ of all samples with relatively small values of $|\bar{X}-\mu| / S$, then the "null hypothesis" that the true mean equals 0 is incorrect.

The probabilistic nature of each conclusion is clear from the stated assumption, and the specific numerical values directly reflect the sample data.

Despite our major disagreements with HMRW, there is certainly support for their overall thesis of confusion regarding the correct interpretation of CIs (see also Fidler 2006, Kalinowski 2010; but see Coulson, Healey, Fidler and
Cumming 2010, for evidence that CIs can facilitate interpretations under some conditions). This thesis is evidently supported to some extent by the fact that participants disagreed with one another about the statements in Table 1; if there were no confusion, participants might have been expected to agree with one another. Indeed, it is further supported by the fact that we have written this reply to HMRW-because obviously we disagree with them. Their thesis is also supported by the finding that many participants endorsed statement 6 , because these endorsements suggest the participants mistakenly thought that the true mean is a random variable. From our point of view, the thesis is also supported to some degree by HMRW's finding that only about half of the researchers endorsed statement 5 . Though HMRW regard that statement as false and think that fewer should have endorsed it, we regard the statement as true and think more should have endorsed it. Ultimately, though, it is impossible to draw any firm conclusion from the proportion of researchers who endorsed this statement because of the ambiguity of the critical term "confidence."

In the final analysis, we suspect that researchers' endorsements of the statements in Table 1 have little bearing on their ability to draw conclusions from CIs within actual research settings. If there truly are serious practical misunderstandings about CIs, then it should be possible to find cases in which misinterpretation of CIs led to incorrect substantive conclusions with damaging consequences for research. To our knowledge, this has never been done. Thus, although better statistical education is always a worthwhile goal, it seems premature to suggest that CIs are completely misunderstood and that a technique with solid mathematical foundations should therefore be abandoned (Morey et al., 2014).

Author Note The authors thank Gregory Francis, Jürgen Heller, Rink Hoekstra, Caitlin Miller, Dirk Vorberg, and especially Wolf Schwarz for helpful comments on earlier versions of the article.

\section{References}

Carpenter, S. (2012). Psychology's bold initiative. Science, 335(6076), 1558-1561. doi:10.1126/science.335.6076.1558

Chatterjee, S. K. (2003). Statistical thought: A perspective and history. Oxford: Oxford University Press.

Coulson, M., Healey, M., Fidler, F., \& Cumming, G. (2010). Confidence intervals permit, but don't guarantee, better inference than statistical significance testing. Frontiers in Psychology, 1(26). doi:10.3389/fpsyg.2010.0002

Cumming, G., \& Finch, S. (2005). Inference by eye: Confidence intervals and how to read pictures of data. American Psychologist, 60, 170-180. doi:10.1037/0003-066X.60.2.170

DeGroot, M. H. (1989). Probability and statistics, 2nd edition. Menlo Park: Addison-Wesley. 
Dempster, A. P. (1964). On the difficulties inherent in Fisher's fiducial argument. Journal of the American Statistical Association, 59(305), 56-66. doi:10.2307/2282858

Dracup, C. (2005). Confidence intervals. In B. S. Everitt, \& D. C. Howell (Eds.), Encyclopedia of statistics in behavioral science, (Vol. 1 A-D pp. 366-375): Wiley.

Fidler, F. (2006). From statistical significance to effect estimation: Statistical reform in psychology, medicine and ecology. $\mathrm{PhD}$ thesis: University of Melbourne.

Francis, G. (2013). Replication, statistical consistency, and publication bias. Journal of Mathematical Psychology, 57(5), 153-169. doi:10.1016/j.jmp.2013.02.003 Special Issue: A Discussion of Publication Bias and the Test for Excess Significance.

Gilliland, D., \& Melfi, V. (2010). A note on confidence interval estimation and margin of error. Journal of Statistics Education, 18(1), $1-8$.

Hájek, A. (2012). Interpretations of probability. In E. N. Zalta (Ed.), The Stanford Encyclopedia of Philosophy, Winter 2012 edition. http://plato.stanford.edu/archives/win2012/entries/ probability-interpret/

Hays, W. L. (1973). Statistics for the social sciences. New York: Holt, Rinehart, \& Winston.

Hoekstra, R., Morey, R. D., Rouder, J. N., \& Wagenmakers, E.-J. (2014). Robust misinterpretation of confidence intervals. Psychonomic Bulletin \& Review, 21(5), 1157-1164. doi:10.3758/s13423-013-0572-3

Hogg, R. V., \& Craig, A. T. (1970). Introduction to mathematical statistics, 3rd edition. New York: Macmillan.

Ioannidis, J. P. A. (2005). Why most published research findings are false. PLoS Medicine, 2, 696-701. doi:10.1371/journal.pmed.0020124

Kalinowski, P. (2010). Identifying misconceptions about confidence intervals. In Proceedings of the eighth international conference on teaching statistics. Lijbljana.

Keren, G. (1991). Calibration and probability judgements: Conceptual and methodological issues. Acta Psychologica, 77(3), 217-273. doi:10.1016/0001-6918(91)90036-Y
Mood, A. M., Graybill, F. A., \& Boes, D. C. (1974). Introduction to the theory of statistics, 3rd edition. New York: McGraw-Hill.

Morey, R., Wagenmakers, E.-J., Hoekstra, R., Rouder, J., \& Lee, M. D. (2014). The fallacy of placing confidence in confidence intervals. Presentation at the annual meeting of the Psychonomic Society, Long Beach, Nov.

Neyman, J. (1937). Outline of a theory of statistical estimation based on the classical theory of probability. Philosophical Transactions of the Royal Society of London. Series A, Mathematical and Physical Sciences, 236(767), 333-380.

Open Science Collaboration (2012). An open, large-scale, collaborative effort to estimate the reproducibility of psychological science. Perspectives on Psychological Science, 7(6), 657-660. doi: $10.1177 / 1745691612462588$

Pashler, H., \& Harris, C. (2012). Is the replicability crisis overblown? Three arguments examined. Perspectives on Psychological Science, 7(6), 531-536. doi:10.1177/1745691612463401

Robinson, G. K. (1975). Some counterexamples to the theory of confidence intervals. Biometrika, 62(1), 155-160.

Robinson, G. K. (1982). Confidence intervals and regions. In S. Kotz, \& N. L. Johnson (Eds.), Encyclopedia of statistical sciences, (Vol. 2 pp. 120-127): Wiley.

Savage, L. J. (1954). The foundations of statistics: Wiley.

Simmons, J. P., Nelson, L. D., \& Simonsohn, U. (2011). False-positive psychology: Undisclosed flexibility in data collection and analysis allows presenting anything as significant. Psychological Science, 22(11), 1359-1366. doi:10.1177/0956797611417632

Simonsohn, U., Nelson, L. D., \& Simmons, J. P. (2014). P-curve: A key to the file-drawer. Journal of Experimental Psychology: General, 143(2), 534-547. doi:10.1037/a0033242

Von Mises, R. (1957). Probability, statistics, and truth. London: George Allen \& Unwin.

Wilkinson, L., \& the Task Force on Statistical Inference (1999). Statistical methods in psychology journals: Guidelines and explanations. American Psychologist, 54(8), 594-604. doi:10.1037//0003-066X.54.8.594 